\title{
Summary of the best evidence for postural change in the prevention of pressure injury in critically ill adult patients
}

\author{
Jin Xue ${ }^{1,2,3 \#}$, Xiao Gu ${ }^{2,4 \#}$, Ling Jiang ${ }^{2,5}$, Niankai Cheng ${ }^{2,5}$, Qin Huang ${ }^{2,6}$, Min Wang ${ }^{2,4}$ \\ ${ }^{1}$ Department of EICU, Suzhou Municipal Hospital, The Affiliated Suzhou Hospital of Nanjing Medical University, Suzhou, China; ${ }^{2}$ Gusu College \\ of Nanjing Medical University, Suzhou, China; ${ }^{3}$ School of medicine, Jiangsu university, Zhenjiang, China; ${ }^{4}$ Department of ICU, Suzhou Municipal \\ Hospital, The Affiliated Suzhou Hospital of Nanjing Medical University, Suzhou, China; ${ }^{5}$ Department of Nursing, Suzhou Municipal Hospital, \\ The Affiliated Suzhou Hospital of Nanjing Medical University, Suzhou, China; ${ }^{6}$ Department of Emergency and Critical Care, Suzhou Municipal \\ Hospital, The Affiliated Suzhou Hospital of Nanjing Medical University, Suzhou, China \\ Contributions: (I) Conception and design: J Xue, X Gu; (II) Administrative support: L Jiang; (III) Provision of study materials or patients: N Cheng; (IV) \\ Collection and assembly of data: Q Huang; (V) Data analysis and interpretation: M Wang; (VI) Manuscript writing: All authors; (VII) Final approval \\ of manuscript: All authors. \\ \#These authors contributed equally to this work. \\ Correspondence to: Min Wang. Department of ICU, Suzhou Municipal Hospital, The Affiliated Suzhou Hospital of Nanjing Medical University, Gusu \\ College of Nanjing Medical University, Suzhou 215000, China. Email: wangmin_19833@163.com.
}

\begin{abstract}
Background: This study sought to evaluate and summarize the best evidence on postural change in the prevention of pressure injuries among critically ill adult patients, to provide a reference for the treatment of these patients in clinical practice.

Methods: An evidence-based approach was adopted to retrieve relevant articles from databases and professional websites. The Joanna Briggs Institute evidence appraisal and recommendation system was used to assess the quality and level of the evidence.

Results: In total, 11 articles were included in the analysis, including 4 clinical practice guidelines, 2 expert consensus papers, 1 evidence summary, 3 systematic reviews, and 1 randomized controlled trial. 14 pieces of evidence on the feasibility and suitability of specific clinical situations were summarized, and the following 7 aspects were considered: (I) individual assessment; (II) postural change frequency; (III) postural change angle; (IV) postural change strategies for critically ill patients with hemodynamic instability; (V) assessment during postural change, (VI) multidisciplinary team cooperation; and (VII) education and training.

Conclusions: Clinical nurses should pay attention to the prevention and management of pressure injuries in critically ill adult patients. The approach should be selected and applied according to the specific clinical situation and the individual situation of each patient to achieve better treatment effect.
\end{abstract}

Keywords: Severely ill; postural change; pressure injury; prevention; evidence-based nursing

Submitted Aug 05, 2021. Accepted for publication Nov 10, 2021.

doi: 10.21037/apm-21-2521

View this article at: https://dx.doi.org/10.21037/apm-21-2521

\section{Introduction}

Patients in intensive care units (ICU) are in a critical condition, and often have multiple organ dysfunction, and require long-term bed rest. The incidence of pressure injury in ICU patients is much higher than that of general hospitalized patients. Lahmann et al. (1) analyzed secondary data and found that the possibility of a pressure injury in ICU patients abroad was 4.3 times higher than that of patients in ordinary wards, which was up to $14.9 \%$. A domestic multicenter investigation (2) showed that the incidence of pressure injury in adult ICU patients was $11.92 \%$. The pressure injury is divided into four stages: Stage I (erythema, slight irritation), Stage II (skin 
breakdown, redness and swelling), Stage III (full-thickness skin loss) and Stage IV (exposed bone, muscle, or implant, and infection leading to the removal of the implant) (3). Postural change is performed to prevent the occurrence of pressure injury. When the pressure injury is formed, no matter what the stage is, the injury site should not be pressed any more. As pressure injuries may aggravate the condition of ICU patients, prolong the length of hospitalization, lead to a decline in quality of life (4), and increase of hospitalization costs (5), effective measures must be implemented to prevent them.

At present, there are differences in the selection of evaluation tools for pressure injuries in critically ill adult patients at home and abroad (6), and the frequency and angle of postural changes have not been clearly defined, which has led to differences between clinical practices and existing evidence. In previously study, prone body position has also been applicated to improve respiratory function of acute respiratory distress syndrome (ARDS) patients (7). However, the study was published in 2002 and the patients is restricted to ARDS patients. The postural change in the prevention of pressure injury in critically ill adult patients should be further clarified. In this study, evidence of postural changes in critically ill adult patients was retrieved and the quality of this evidence was evaluated. This study sought to summarize the best evidence on postural change to prevent pressure injuries, provide a relevant decisionmaking reference for clinical nursing staff, reasonably reduce the incidence of pressure injury among critically ill adult patients, improve the clinical outcomes of patients, and improve the quality of care provided to patients.

\section{Methods}

\section{Research problem}

The problem development tool of the Fudan University Evidence-based Nursing Center was adopted to address the initial problem of evidence-based nursing. Using the PICOS principle, the problem was defined as follows: target population of evidence application $(\mathrm{P})$-critically ill adult patients; interventions (I) - postural changes in critically ill adult patients based on best evidence; outcome indicator $(\mathrm{O})$-incidence of pressure injury; type of evidence (S)—clinical decisions, practice guidelines, evidence summaries, expert consensus papers, systematic reviews, and original research closely related to the topic of this study, etc.

\section{Evidence retrieval}

The following Chinese search terms were used: "serious illness/intensive care unit (ICU)/high risk, turn over/ body position change/postural change/reduce pressure/ precaution/Nursing, Pressure injury/pressure sores/ pressure ulcer/deep tissue injury". The following English search terms were used: "Critically ill/intensive care unit/ ICU/risk/high risk, repositioning/turning, Pressure injury/ pressure ulcer/pressure sores/bed sores/decubitus ulcers/ deep tissue injury/prevention/". According to the "6S" pyramid model (8), a top-down retrieval strategy was adopted for the literature retrieval. The following databases and websites were searched: (I) Guideline database websites, including Cochrane Library, CINAHAL, Joanna Briggs Institute (JBI) Database of Evidence-based Health Care Center, BMJ Best Practice, UpToDate, Canadian Medical Association: Clinical Practice Guideline, CMA Infobase (CPG), NICE: National Institute for Health and Care Excellence, Registered Nurses' Association of Ontario (RNAO) and Guideline Website of Medlive.cn; (II) Websites of professional societies, including National Pressure Ulcer Advisory Panel (NPUAP), European Pressure Ulcer Advisory Panel (EPUAP); and (III) Other databases, including PubMed, Embase, China National Knowledge Internet (CNKI), Wanfang database, China Biology Medicine disc (CBM). Articles on pressure injuries in critically ill adult patients were retrieved. The search period ran from the establishment of each database to March 10, 2021.

\section{Literature inclusion and exclusion criteria}

To be eligible for inclusion in this study, the articles had to meet the following inclusion criteria: (I) the subjects comprised critically ill adult patients; (II) the object was to examine the prevention of pressure injuries by postural change; (III) the outcome indicators included the incidence of pressure injury and complications; (IV) the article was a guideline, evidence summary, expert consensus document, systematic evaluation document, original research study, etc.; and $(\mathrm{V})$ the article was in Chinese or English.

Articles were excluded from the study if they met any of the following exclusion criteria: (I) used old versions of guidelines; (II) the document was a research proposal, report, or abstract; (III) the full text could not be obtained; and/or (IV) the article failed to meet the quality evaluation criteria. 


\section{Evidence evaluation criteria}

The guidelines were evaluated using the United Kingdom 2012 update of the clinical guidelines for research and evaluation system II (AGREE II) (9). Six aspects were evaluated, including the scope and purpose, the personnel involved, and the preciseness of the developed guidelines. Each item in each guideline was scored independently in accordance with the above 6 aspects. Finally, the score of each item was standardized according to the formula; the standardized results are presented in the form of a percentage. Under the guidelines, there were three levels: Grade A was awarded if all 6 aspects of the guidelines scored $\geq 60 \%$; Grade B was awarded if $\geq 3$ aspects scored $\geq 30 \%$ but $<60 \%$; Grade $\mathrm{C}$ was awarded if $\geq 3$ aspects scored $<30 \%$. The systematic evaluation was carried out using a systematic review evaluation tool (i.e., AMSTAR 2) (10). The expert consensus papers, randomized controlled trial (RCT), and other articles were evaluated using the evaluation criteria [2016] of the Australian JBI Evidencebased Health Care Center (11). The quality evaluation of the evidence summary was traced back to the original study and an appropriate evaluation criterion was selected according to the literature type.

\section{Literature quality evaluation process}

The included articles were independently evaluated by two researchers trained in systematic evidence-based medicine. In case of disagreement, another member of the inhospital evidence-based care team adjudicated. In case of conflicting conclusions from different sources of evidence, the principle of inclusion in this study was that evidencebased evidence was preferred, high-quality evidence was preferred, and the latest most authoritative literature was preferred.

\section{Level of evidence evaluation}

The JBI evidence pre-grading system (2014 edition) (12) was used to pre-grade the included studies, and the evidence was divided into levels $1-5$. After the pre-grading of evidence, the recommendation level of the evidence was determined according to the feasibility, suitability, clinical significance, and effectiveness of the evidence under the guidance of the JBI FAME structure, combined with the recommendation level of the JBI Levels of Evidence 2014.

\section{Results}

\section{Literature retrieval results}

A total of 320 related articles were retrieved following the preliminary search, and 31 duplicate articles were excluded. After reading the titles and abstracts, 105 articles with population/disease factor incompatibility, 85 articles with intervention method incompatibility, 81 articles with study design/type incompatibility were excluded, and 11 articles remained. After reading the full texts, 11 articles were finally included, including 4 guidelines (13-16), 2 expert consensus papers $(17,18), 1$ evidence summary (19), 3 systematic reviews (20-22), and 1 RCT (23). The general details of the included articles are set out in Table 1 .

\section{Literature quality evaluation results}

\section{Results of quality evaluation of the guidelines}

A total of 4 guidelines were included (13-16), and the evaluation results for these guidelines are set out in Table 2.

\section{Results of quality evaluation of the expert consensus papers}

A total of 2 expert consensus papers were included $(17,18)$, of which 1 (17) was from PubMed and the other 1 (18) was from the Administrative Management Professional Committee of Chinese Nursing Association. The quality of the expert consensus papers was good, and the evaluation results of both articles were "yes".

\section{Results of quality evaluation of systematic reviews}

A total of 3 systematic reviews (20-22) and 2 metaanalyses were included. In the study of Li et al. (20) and Ma et al. (21), the evaluation results for Item 7, "Whether the list of excluded literatures and the reasons for exclusion are provided," Item 10, "Whether the authors of the systematic review reported on the sources of funding for each included study," and Item 16, "Whether the authors of the systematic review have reported all sources of potential conflicts of interest, including any funding received for conducting this review," were "No". The evaluation results of Item 12, "When interpreting/discussing the results of the systematic review, whether the authors considered the risk of bias in the included study," and Item 15, "If quantitative synthesis was performed, whether the authors have adequately investigated publication bias and discussed its possible influence on the study results," were "partly yes". The 
Table 1 Characteristics of included articles

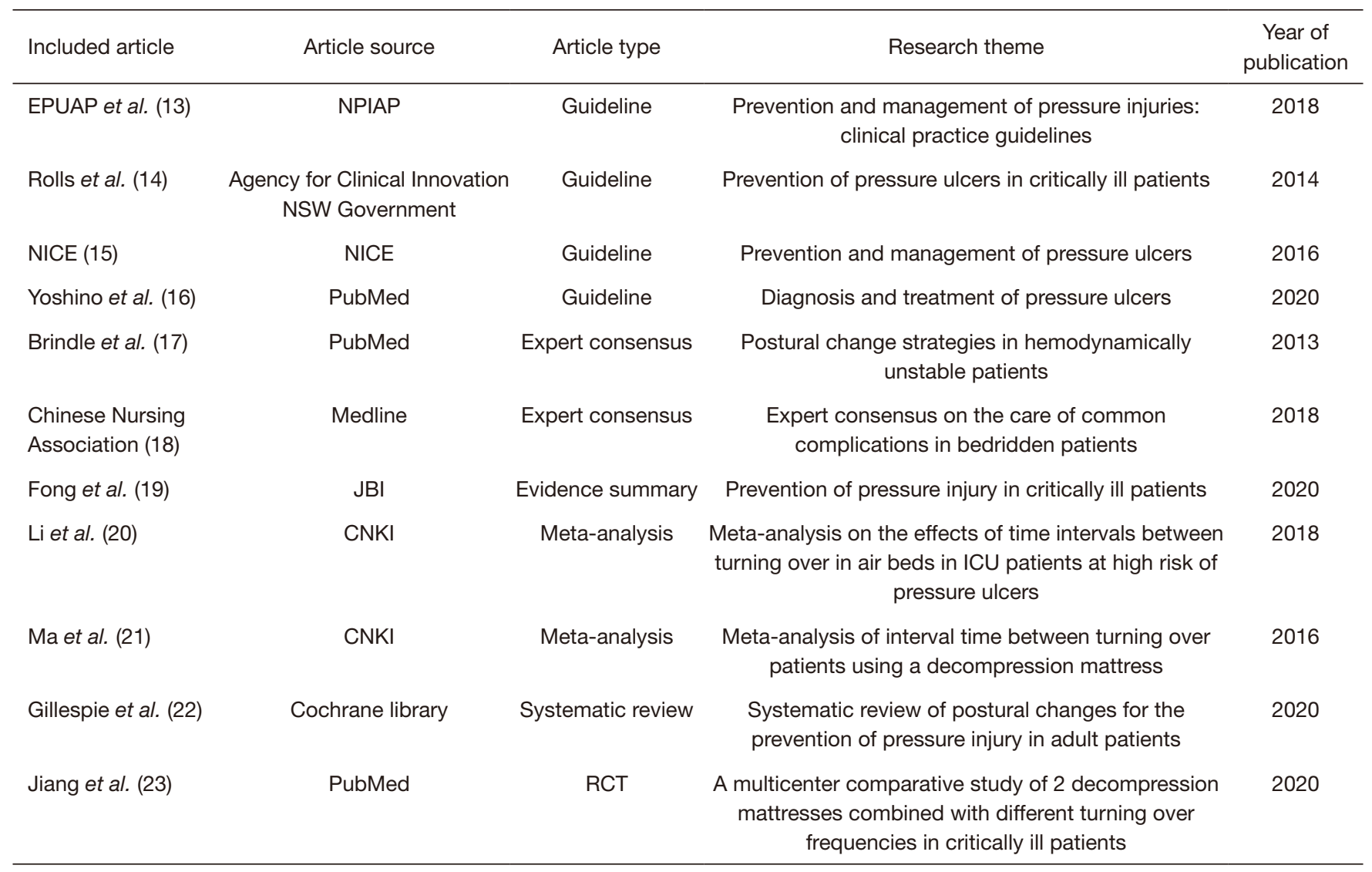

EPUAP, European Pressure Ulcer Advisory Panel; NPIAP, National Pressure Injury Advisory Panel; NICE, National Institute for Health and Care Excellence; $\mathrm{RCT}$, randomized controlled trial.

remaining item results were all "yes". These studies had a high overall quality and were approved for inclusion. 1 systematic review (22) had no data consolidation and metaanalysis. In this systematic review, only Item 11, "Whether to state the relevant conflict of interest, potential sources of resources included in the systematic review and study should be clearly identified," was assessed as "No"; however, the rest of the items were assessed as "Yes", and approved for inclusion.

\section{Results of the quality evaluation of evidence summary and original research}

The quality evaluation of the 1 included evidence summary (19) and the quality evaluation of the 1 included RCT (23) were carried out using the JBI evaluation criteria [2016] (11). The quality evaluation showed that the evaluation results for all the items were, "Yes". The overall quality evaluation was high, and the inclusion of this evidence summary was approved.

\section{Summary of evidence}

We summarized the evidence for postural changes in the prevention of pressure injuries. In this study, evidence was summarized in relation to 7 aspects; that is, individual evaluation, postural change frequency, postural change angle, postural change strategy for critically ill patients with hemodynamic instability, evaluation during postural change, multidisciplinary team cooperation, and education and training, and 14 best pieces of evidence were formed. The evidence content, evidence level, and recommendation level are shown in Table 3.

\section{Discussion}

\section{The scientific nature of evidence summary}

In this study, we systematically searched evidence resources on the prevention of pressure injury by body postural changes in critically ill adult patients, and ultimately 


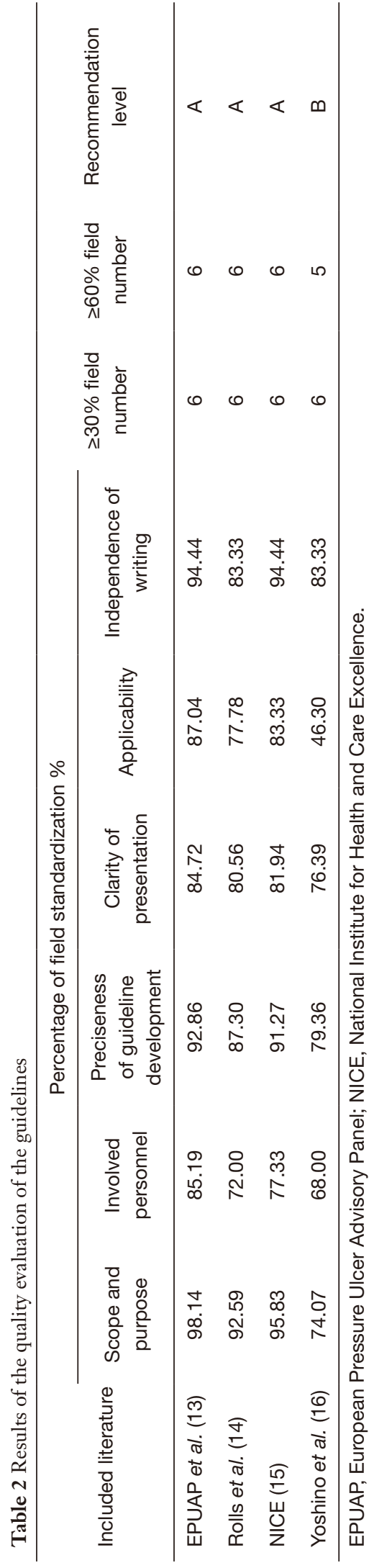

analyzed 11 articles. Through an evidence pre-grading and recommendation level system, the strength of evidence and recommendation level were determined by group discussion. The whole process strictly adhered to the principles of evidence-based nursing to ensure the scientific nature and rigor of the research results.

\section{Applicability of evidence summary}

\section{Risk factors for pressure injury in critically ill adult patients}

Articles 1-3 summarized evidence on individualized assessments before postural changes. The relevant evidence was mainly derived from guidelines (11-14), an expert consensus paper (16), an evidence summary (17), systematic reviews (18-20), and a RCT (21). As the occurrence of pressure injury is a process of physiological changes under the combined action of multiple factors, a large number of original and secondary studies have examined the effects of patients' autonomous activities, mobility, existing or previous stress injuries, pain, local skin tissue perfusion, circulation, oxygenation, moisture, temperature, systemic nutritional status, sensory ability, old age, blood test results, demographic characteristics, systemic, and local decompression measures on the incidence of pressure injuries. The comprehensive assessment of influencing factors is deficient in feasibility and applicability. The Braden Scale, which is the most widely used risk assessment tool for pressure injury, has been proven to be reliable in the assessment of pressure injuries in critically ill patients, but it was not specifically developed for such patients.

\section{Frequency and angle of postural changes in critically ill adult patients}

The evidence in articles 4-5 summarized the frequency of patient postural changes, and the relevant evidence was mainly derived from guidelines (11-14), expert consensus papers (16), systematic reviews (18-20), and an RCT (21). A summary of the evidence published by JBI in 2020 recommended that postures be changed every 2 hours in critically ill adult patients. Multiple meta-analyses showed that the postural change interval could be extended to 4 hours in critically ill adult patients when combined with dynamic and static whole-body decompression tools, such as high-standard foam mattresses and alternate air mattresses.

Articles 6-7 summarized the angle requirements for postural changes, and relevant evidence was mainly derived from the guidelines (11-14), an expert consensus paper (16), 
Table 3 Summary of the best evidence for repositioning in adult intensive patients

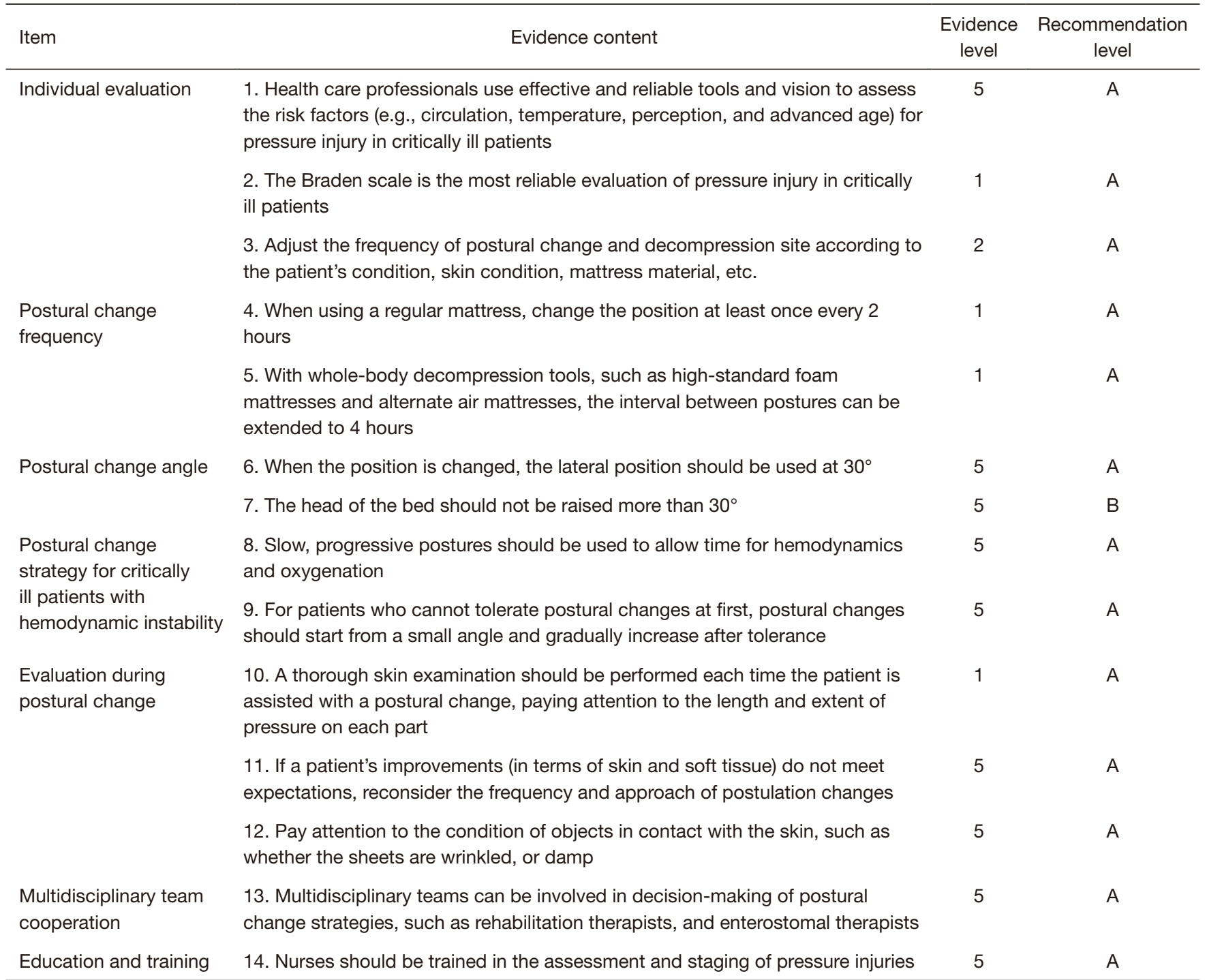

and a systematic review (20). Some studies reported on the effects of lateral sleeping at $30^{\circ}$ and $45^{\circ}$ on pressure injuries in critically ill patients. The quality of the studies was low due to the sample sizes and unclear reporting of "routine care". Other studies compared the angle of postural change at $30^{\circ}$ and $90^{\circ}$, mostly in elderly patients, but their findings have little relevance for critically ill patients. In relation to bed head elevation, minimizing the headboard height in the decumbent position significantly reduces friction and shear forces, as well as the associated risk of pressure damage. However, for critically ill patients, raising the head of the bed was also related to the prevention of reflux and aspiration, ventilator-associated pneumonia, etc. In practice, the position should be adjusted and the angle changed to ensure that patients do not easily slip or become deformed. Additionally, attempts should be made to distribute the pressure and friction over a large area.

Articles 8-9 summarized postural change strategies for critically ill patients with unstable vital signs, and the relevant evidence was mainly derived from an expert consensus paper (15). The expert consensus paper recommended that nurses follow the procedure of postural change and adopt a slow and gradual postural change strategy when treating patients with hemodynamic instability. The main operation steps are to turn the body every $10-15^{\circ}$, monitor the patient's vital signs for 15 
seconds, and if the vital signs are stable, continue to turn the body until the required angle by the nursing operation is reached. The nurses should use the same 15 -second rotation $15^{\circ}$-strategy to return to $30^{\circ}$, and continue to observe vital signs for 10 minutes using a triangular pillow, soft pillow, etc. For patients who cannot tolerate postural changes at the beginning, the postural changes should start at a small angle and gradually increase as tolerance increases.

\section{Evaluation of skin and skin contact sites during postural changes in critically ill adult patients}

Articles 10-12 summarized relevant evidence on the evaluation of skin and skin contact sites during postural changes. The evidence was mainly derived from guidelines (11-14) and an expert consensus paper (16). Nurses observe and record the condition and response of compression sites while assisting patients to change position. If a patient's improvements (in terms of skin and soft tissue) do not meet expectations, the frequency and approach of postural changes should be reconsidered.

\section{Multidisciplinary cooperation}

Article 13 summarized the evidence related to the postural change strategy of multidisciplinary teams involved in decision making. The evidence was mainly derived from guidelines (11-14) and an expert consensus paper (16). A multidisciplinary team, including doctors, nurses, rehabilitation therapists and ana enterostomal therapists, were involved in determining the postural change strategy.

\section{Strengthen personnel training}

Article 14 was an evidence guide (11) that recommended that nursing staff be trained in the international pressure sore staging system and medical professionals be trained to undertake accurate and reliable risk assessments. Only by improving nurses' awareness of the assessment of risk factors of pressure injury and early identification of high-risk patients can we ensure the implementation of preventive measures and thus reduce the incidence of pressure injury in critically ill adult patients.

In total, seven aspects of postural change should be notice to perform good postural change: (I) individual assessment; (II) postural change frequency; (III) postural change angle; (IV) postural change strategies for critically ill patients with hemodynamic instability; (V) assessment during postural change, (VI) multidisciplinary team cooperation; and (VII) education and training.

\section{Limitations of this evidence summary}

This evidence summary included only published clinical guidelines, evidence summaries, expert consensus, systematic reviews, and original studies in both Chinese and English. The articles in other languages could be included to form better evidence summary. The comprehensiveness of this study search may be lacking and this study does not include device-related pressure injuries. Thus, in further studies, other etiologies induced pressure injury could be included. The entries in this evidence summary were from abroad. In the process of evidence application, clinical context needs to be further considered to develop localized practice plans. Future clinical reviews will be carried out to assess any obstacles to the application of the evidence.

\section{Conclusions}

In the clinical practice, the adverse consequences of pressure injury injuries to in adult critically ill patients critically ill adult patients have been deeply generally accepted, and various measures to reduce pressure injuries have been widely used. However, nursing staff often lack an understanding for of the most basic turning and posture postural change measures, nursing staff often lack of understanding. On the one hand, indeed, nursing staff believe that the higher grater the frequency of the postural change, the better the effect that postural change has in of preventing pressure injuries. However, this ignores the impact effects of excessive turning on patients' sleep, and nurses' workloads. On the other hand, incorrect posture postural changes do not have a preventive effect, and may even have the opposite effect. The aim of this study was to give evidence-based strategy to standardized the method of postural change. This study summarized the current evidence on the prevention of pressure injury by postural change in adult critically ill patients, and provides a reference for clinical medical staff and medical decision makers to standardize operation procedures.

\section{Acknowledgments}

Funding: None.

\section{Footnote}

Conflicts of Interest: All authors have completed the ICMJE uniform disclosure form (available at https://dx.doi. 
org/10.21037/apm-21-2521). The authors have no conflicts of interest to declare.

Ethical Statement: The authors are accountable for all aspects of the work in ensuring that questions related to the accuracy or integrity of any part of the work are appropriately investigated and resolved.

Open Access Statement: This is an Open Access article distributed in accordance with the Creative Commons Attribution-NonCommercial-NoDerivs 4.0 International License (CC BY-NC-ND 4.0), which permits the noncommercial replication and distribution of the article with the strict proviso that no changes or edits are made and the original work is properly cited (including links to both the formal publication through the relevant DOI and the license). See: https://creativecommons.org/licenses/by-nc-nd/4.0/.

\section{References}

1. Lahmann NA, Kottner J, Dassen T, et al. Higher pressure ulcer risk on intensive care? - comparison between general wards and intensive care units. J Clin Nurs 2012;21:354-61.

2. Xu L, Jiang Q. Research of Prevalence of Pressure Ulcer and Hospital-acquired Pressure Ulcer. Journal of Nursing (China) 2012;19:09-5.

3. Hsieh HS, Lee CY, Wu HP, et al. Data for pressure ulcers and skin infections after cochlear implantation. Data Brief 2020;32:106295.

4. Gorecki C, Nixon J, Madill A, et al. What influences the impact of pressure ulcers on health-related quality of life? A qualitative patient-focused exploration of contributory factors. J Tissue Viability 2012;21:3-12.

5. Dealey C, Posnett J, Walker A. The cost of pressure ulcers in the United Kingdom. J Wound Care 2012;21:261-2, 264, 266.

6. Huang C, Ma Y, Jiang M, et al. Research Progress of Stress Injury Risk Assessment Tools. Shanghai Nursing, January 2021;11:50-3.

7. Pelosi P, Brazzi L, Gattinoni L. Prone position in acute respiratory distress syndrome. Eur Respir J 2002;20:1017-28.

8. Dicenso A, Bayley L, Haynes RB. Accessing pre-appraised evidence: fine-tuning the $5 \mathrm{~S}$ model into a $6 \mathrm{~S}$ model. Evid Based Nurs 2009;12:99-101.

9. Hoffmann-Eßer W, Siering U, Neugebauer EA, et al. Guideline appraisal with AGREE II: Systematic review of the current evidence on how users handle the 2 overall assessments. PLoS One 2017;12:e0174831.

10. Shea BJ, Reeves BC, Wells G, et al. AMSTAR 2: a critical appraisal tool for systematic reviews that include randomised or non-randomised studies of healthcare interventions, or both. BMJ 2017;358:j4008.

11. Hu Y, Hao Y. Evidence-Based Nursing. Beijing: People's Medical Publishing House, 2019:52-106.

12. Wang C, Hu Y. JBI Evidence Pre-Grading and Evidence Recommendation Level System (2014 edition). Journal of Nurses Training 2015;30:964-7.

13. European Pressure Ulcer Advisory Panel, National Pressure Ulcer Advisory Panel, Pan Pacific Pressure Injury Alliance. Prevention and Treatment of pressure ulcers/ injuries: methodology Protocol for the clinical practice guideline (third ed.). EPUAP, NPUAP, PPPIA. 2018 May:51-8.

14. Rolls K, Jones S, Power D, et al. Pressure injury prevention for critically ill adults: a clinical practice guideline. Agency for Clinical Innovation NSW Government Version 1, Chatswood NSW 2014:ISBN 978-1-74187-955-1.

15. Proceedings of the 3rd IPLeiria's International Health Congress : Leiria, Portugal. 6-7 May 2016. BMC Health Serv Res 2016;16 Suppl 3:200.

16. Yoshino Y, Hashimoto A, Ikegami R, et al. Wound, pressure ulcer and burn guidelines - 6: Guidelines for the management of burns, second edition. J Dermatol 2020;47:1207-35.

17. Brindle CT, Malhotra R, O'rourke S, et al. Turning and repositioning the critically ill patient with hemodynamic instability: a literature review and consensus recommendations. J Wound Ostomy Continence Nurs 2013;40:254-67.

18. Project Team of the Construction of Standardized Nursing Intervention Model for the Common Complications among Bedridden Patients, Administrative Committee of Chinese Nursing Association. A consensus statement on nursing care of major immobility complications among bedridden patients. Chinese Nursing Management 2018;18:740-7.

19. Fong E. Evidence Summary. Pressure Injury (Critically Ill Patients): Prevention. The JBI EBP Database 2020:JBI24357.

20. Li F, Yan L, Song M, et al. Influence of turn over interval of air cushion bed on high rish pressure ulcer patients in ICU: a meta-analysis. Journal of Nursing Science 2018;33:95-8.

21. Ma D, Ding P, Li H. A Meta Analysis of Repositinging 
Schedules for Patients Using Decompression Mattresses. Journal of Nursing Science 2016;31:99-103.

22. Gillespie BM, Walker RM, Latimer SL, et al. Repositioning for pressure injury prevention in adults. Cochrane Database Syst Rev 2020;6:CD009958.

23. Jiang Q, Liu Y, Yu H, et al. A Multicenter, Comparative

Cite this article as: Xue J, Gu X, Jiang L, Cheng N, Huang Q, Wang M. Summary of the best evidence for postural change in the prevention of pressure injury in critically ill adult patients. Ann Palliat Med 2021;10(11):11445-11453. doi: 10.21037/apm-212521
Study of Two Pressure-Redistribution Mattresses with Repositioning Intervals for Critical Care Patients. Adv Skin Wound Care 2020;33:1-9.

(English Language Editor: L. Huleatt) 